\title{
Possible Connection between High-Voltage Power Lines \& Cancer
}

Paul T E Cusack

BScE, DULE, 23 Park Ave. Saint John, NB, E2J 1R2, Canada

*Corresponding Author: Paul T E Cusack, BScE, DULE, 23 Park Ave. Saint John, NB, E2J 1R2, Canada.

Received date: August 17, 2020: Accepted date: September 25, 2020: Published date: September 30,2020

Citation: Paul T E Cusack (2020) Possible Connection between High-Voltage Power Lines \& Cancer. J General Medicine and Clinical Practice. 3(3) DOI: $10.31579 / 2639-4162 / 031$

Copyright: (C2020. Paul T E Cusack This is an open-access article distributed under the terms of the Creative Commons Attribution License, which permits unrestricted use, distribution, and reproduction in any medium, provided the original author and source are credited.

\section{Abstract \\ We have heard for years that there may be a connection between high voltage lines and cancer. Since my previous paper on Hydrogen Peroxide as a carcinogen, coupled with my equations of the mind, soul, and consciousness, I purpose a mathematical connection between Power lines and cancer. There should be safe distance to keep the magnetic field produced by the lines and the mind. \\ Keywords: high voltage power line; cancer; hydrogen peroxide; at math; mind; soul; inductor}

\section{Introduction}

The brain has a ongoing "movie" of images and language in the imagination. The imagination is an electro-magnetic field produced by the mind which is an inductor ( $\mathrm{L}=2$ Henries). We have seen from a previous paper that the voltage across this inductor $=\mathrm{V}=22.3$ and the current through it is $\mathrm{i}=1 / \mathrm{c}^{2}$.

The equation of the mind is:

Soul Energy=Energy -Mind

$=1 / 8-1 . / 9=1 / 720=138.888$

The basic electrical equation is $\mathrm{V}=\mathrm{iR}$

$223=\left(1 / \mathrm{c}^{2}\right) \mathrm{R}$

$\mathrm{R}=2.007 \sim 2=\mathrm{L}$

$\mathrm{P}=\mathrm{i}^{2} \mathrm{R}$

$=\left(1 / \mathrm{c}^{2}\right) 2$

$=246.7 \sim 247$

Now consider the power lines. They operate at a voltage of 345,000 Volts

$$
\begin{aligned}
& \mathrm{P}=\mathrm{V} \times \mathrm{A} \\
& 247=345,000 \mathrm{~A} \\
& \mathrm{~A}=715.9 \\
& \sim 716 \\
& \mathrm{P}=\mathrm{i}^{2} \mathrm{R} \\
& 247=716^{2} \mathrm{R} \\
& \mathrm{R}=481.2 \sim 481
\end{aligned}
$$

$$
\begin{aligned}
& V=i R \\
& =716(481) \\
& =3446
\end{aligned}
$$

Eq of the mind/ Power $=138.888 / 246.4=406=1 / 246.3$ $=1 / \mathrm{P}$

Eq. of the Mind/P=1/P

Eq of the Mind $=1$

$V=3446$

Vof the Circuit of the mind $=5444-3446=1998 \sim 2+\mathrm{L}$ 


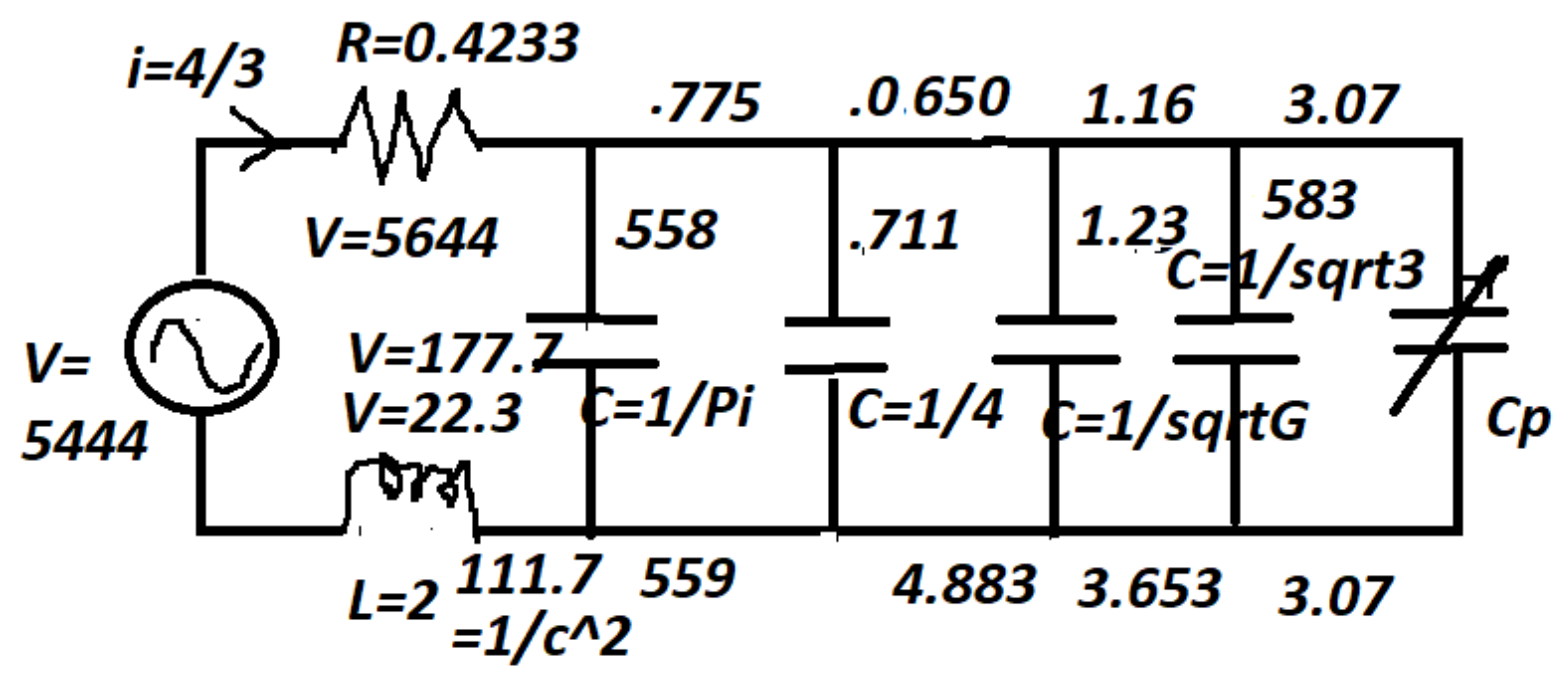

\section{$V=i R \quad 177.7=3.07(R)$ \\ $R=57.88=1 \mathrm{rad} \quad 1 /$ sqrt3 =Universal Signal $C=1 /$ sqrt 3}

\section{Figure 1 Circuit of the Human Senses}

Now consider that Hydrogen Peroxide causes cancer as established by a previous paper of mine. How does $\mathrm{H} 2 \mathrm{O} 2$ figure into the mind equation?

\section{$\mathrm{H}=2 \mathrm{x} 1.008=201.6$}

$\underline{\mathrm{O} 2=2 \times 15.999=31.998}$

$\Sigma=23.35 \mathrm{gm} / \mathrm{mol} \times 6.023 \mathrm{moles}=140.69 \mathrm{gm}=$ Mass M

$\mathrm{E}=(1-\operatorname{Ln} \mathrm{t})^{7}$

$=(1-\operatorname{Ln} 140.69)^{7}$

$=724.6$

$1 / \mathrm{E}=\mathrm{t}=0.000001308$

$\mathrm{t}=\mathrm{KE}=1 / 2 \mathrm{Mv}^{2}$

$=\left(1 / 2(140.69)(1 / \sqrt{ } 2)^{2}\right.$

$=35.225$

$1308 \times 35.225=1 / 217=\mathrm{t}^{2}$

$t=214.7$

$\mathrm{E}=(1-\operatorname{Ln} 214.7)^{7}$

$=304 \sim 305$

$1308 / 35.225=371.3$

$=1 / 267$

$=1 / \Pi$ Senses

$=$ Cusack's 9 th Equation of consciousness from a previous paper.
$\mathrm{TE}=\mathrm{PE}+\mathrm{KE}+\mathrm{SE}$

$\Delta \mathrm{E}=\mathrm{E}-\mathrm{M}$

$1=(1-\operatorname{Ln} 720)-\mathrm{PE} / \mathrm{c}^{2}$

$753-\mathrm{PE} / \mathrm{c}^{2}=1$

$\mathrm{PE}=247 \mathrm{c}^{2}$

$=222$

$E=(1-\operatorname{Ln} t)^{7}$

$(1-0.720)^{7}$

$=752.7$

$\mathrm{t}=\mathrm{KE}=1 / 2 \mathrm{Mv}^{2}$

$=1 / 2(752.7)(1 / \sqrt{ } 2)^{2}$

$=188.175$

$\mathrm{TE}=222+188.2+138.888$

$=327$

$=1 / 305=1 / \mathrm{t}=\mathrm{E}$

Now the Mind $=\mathrm{L}=2$

$\operatorname{Mind}=\Delta \mathrm{E}=1$

$\mathrm{SE}=(1 / 8-1 / 9)=138.8$ 
$138.8 / 2=69.4 \sim 70=\mathrm{mV}=$ Resting Voltage

1/69.4=1441 1/0.693 1/7=Ln 2=Ln L

$138.8 / 2=\operatorname{Ln} 2$

$\mathrm{E}=138.8$

$\mathrm{t}=1 / 138.8=720=1 / \mathrm{Mind}$

$\mathrm{E}=\mathrm{Mind}=1.38 .88$

$\mathrm{SE}=\mathrm{t}^{2}-\mathrm{t}-1$

$=720^{2}-720-1$

$=517.7$

So, $\mathrm{E}=\mathrm{Mind}=138.888$

$\mathrm{SE}=517.7$

1388/517.7=268 $\Pi$ Senses $/ \Sigma$ Senses $=267$ Cusack's 9th Eq. of Consciousness

$\mathrm{t}=\mathrm{KE}=1 / 2 \mathrm{Mv}^{2}$

$=1 / 2(752.7)(1 / 2)$

188.175

$188.175 \times \mathrm{c}^{2}=1693$

$188.175=\mathrm{Ec}=\mathrm{Mc}^{4}$

Mass of $\mathrm{H} 2 \mathrm{O} 2=140.9$

140./9(81)

$=113.985$

But $\mathrm{V}=\mathrm{iR}$

$=(4 / 3)(856)$

$=113.3 \quad$ Cf. 113.9

\section{$V=113.3$}

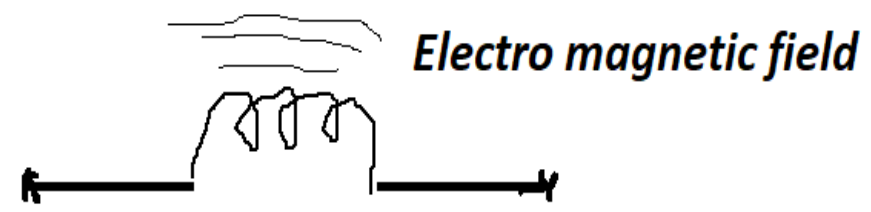

$L=2$
$\mathrm{L}=2 \mathrm{~V}=113.3$

$\mathrm{V}=\mathrm{iR}$

$113.3=\mathrm{i}(2)$

$\mathrm{i}=5665$

$=1 / 176.5 \sim 1 / 177=1 /$ Power

$\mathrm{P}=\mathrm{i}^{2} \mathrm{R}$

$\mathrm{i}=1 /\left(\mathrm{i}^{2} \mathrm{R}\right)$

$\mathrm{i}^{3} \mathrm{R}=1=$ Mind

$(715.9)^{3}(0.856)=3.141 \sim \pi$

Sight $=1 / \pi$

Hearing $=\pi$

The senses pf hearing and sight are dependent upon Pi. This leaves us off where we began.

\section{Conclusion}

We see that $\mathrm{H} 2 \mathrm{O} 2$ which causes cancer is developed from the high voltage lines.

\section{References}

1. Cusack, PTE. Hydrogen Peroxide and Cancer. Lupine, USA. 2018.

2. Dennett., DC. Consciousness Explained. Back Bay Books. NY 1991 